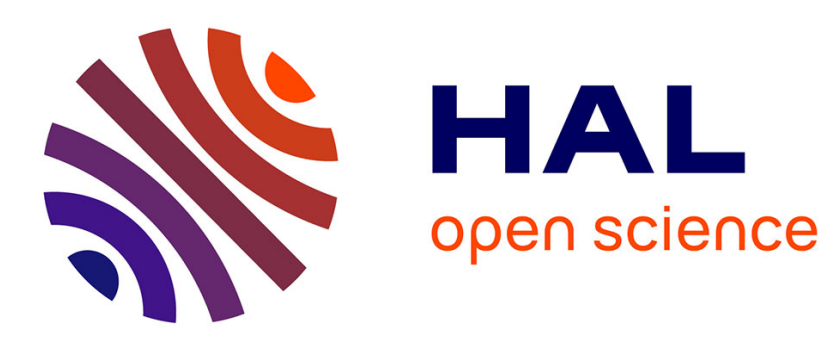

\title{
Static and cyclic stretching: Their different effects on the passive torque-angle curve
}

Antoine Nordez, Peter Mcnair, Pascal Casari, Christophe Cornu

\section{To cite this version:}

Antoine Nordez, Peter Mcnair, Pascal Casari, Christophe Cornu. Static and cyclic stretching: Their different effects on the passive torque-angle curve. Journal of Science and Medicine in Sport, 2010, 13 (1), pp.156-160. 10.1016/j.jsams.2009.02.003 . hal-01007320

\section{HAL Id: hal-01007320 \\ https://hal.science/hal-01007320}

Submitted on 20 Nov 2018

HAL is a multi-disciplinary open access archive for the deposit and dissemination of scientific research documents, whether they are published or not. The documents may come from teaching and research institutions in France or abroad, or from public or private research centers.
L'archive ouverte pluridisciplinaire HAL, est destinée au dépôt et à la diffusion de documents scientifiques de niveau recherche, publiés ou non, émanant des établissements d'enseignement et de recherche français ou étrangers, des laboratoires publics ou privés. 


\title{
Static and cyclic stretching: Their different effects on the passive torque-angle curve
}

\author{
A. Nordez ${ }^{\text {a }}$ P.J. McNair ${ }^{\text {b }}$, P. Casari ${ }^{\text {c }}$, C. Cornu ${ }^{\mathrm{a}, *}$ \\ a Université de Nantes, Nantes Atlantique Universités, Laboratoire "Motricité, Interactions, Performance" (EA 4334), France \\ ${ }^{\mathrm{b}}$ Health and Rehabilitation Research Centre, Auckland University of Technology, New Zealand \\ ${ }^{c}$ Université de Nantes, Nantes Atlantique Universités, CNRS, Institut de recherche en Génie Civil et Mécanique, UMR 6183 France
}

Received 10 December 2007; received in revised form 3 February 2009; accepted 9 February 2009

\begin{abstract}
The findings of previous research indicate that the passive torque-angle curve may be different according to whether individuals have undertaken cyclic or static stretching. To date, no authors have compared these curves in the same subjects. We hypothesised that static stretching would lead to a constant change in range of motion across torque levels with the shape of the curve being unchanged, while cyclic stretching would change the shape of the curve. To test this hypothesis, eight subjects performed five passive knee extension/flexion cycles on a Biodex ${ }^{\circledR}$ dynamometer at $5^{\circ} \mathrm{s}^{-1}$ to $80 \%$ of their maximal range of motion before and after a static stretching protocol. The difference in angle between pre and post stretching torque-angle curves was calculated at 11 levels of torque from $0 \%$ to $100 \%$ of the maximal torque with a $10 \%$ increment. The mean change in angle across these 11 torque levels was then calculated. The findings showed that after static stretching a relatively constant mean change of $5.2^{\circ}$ was noted across torque levels. In contrast, after cyclic stretching the angle change depended upon the torque level with greater change observed toward the start of the range of motion. The findings indicated that different mechanisms were operating depending upon the type of stretching procedure performed. Changes in muscle resting length and thixotropy were thought to be responsible.
\end{abstract}

Keywords: Torque-angle relationship; Passive stretching; Muscle resting length; Creep; Thixotropy

\section{Introduction}

In humans, the passive viscoelastic properties of a musculo-articular complex, including structures spanning the joint,${ }^{1}$ can be determined using passive loading and unloading torque-angle responses. ${ }^{2-11}$ Using these data, many studies have shown that the passive torque and stiffness are altered immediately after cyclic (i.e. dynamic or passive motion) $)^{6,8,9,11}$ and static ${ }^{6-8,10}$ stretching protocols. While these passive stretching exercises are commonly performed in sports and rehabilitation, the mechanisms suggested for such changes are not well known and the acute effects of stretching on the biomechanical properties of a musculo-articular complex remains a topic of continued interest to researchers.

\footnotetext{
* Corresponding author.

E-mail address: christophe.cornu@univ-nantes.fr (C. Cornu).
}

Recent studies $8,11,12$ have provided some indirect evidence that the response of the musculo-articular complex to static and cyclic stretching may be different. After acute static stretching, there appears to be a shift to the right in this relationship indicative of increased range of motion for a particular resistive torque level. This increase in range of motion seems to be constant across all torque levels. In contrast, after cyclic stretching, while a shift to the right is also often evident, the magnitude of change across the range of motion is different, with a greater amount noted in the early part of the range of motion over which the musculo-articular complex is being stretched. Thus for cyclic stretching, the shape of the torque-angle relationship is changed. Should the differences mentioned above be confirmed, the results would indicate that there are different mechanisms involved in these types of stretching procedures. A constant change in range of motion across torque levels is indicative of changes in muscle and tendon length primarily, while variable changes across 
torque levels are more indicative of a thixotropic response. In this instance, thixotropy refers primarily to the damping or viscous responses of the tissues. Such differences may have ramifications for the type of stretches undertaken for rehabilitation or preparation for sports.

Therefore, the purpose of the current study was to confirm the above mentioned qualitative observations and compare the torque-angle relationship before and after an acute bout of cyclic and static stretching. It was hypothesised that cyclic and static stretching protocols could induce different changes in the passive torque-angle curves, and these would be related to changes in muscle resting length or changes in viscous properties. The results of this study will contribute to our understanding of mechanisms related to the acute effects of passive stretching.

\section{Methods}

Eight healthy males $(23.3 \pm 1.9$ years, height: $181.3 \pm 7.0 \mathrm{~cm}$, mass: $74.3 \pm 4.7 \mathrm{~kg}$ ) volunteered to participate in this study and signed an informed consent form. This study was conducted according to the Helsinki Statement (1964) and was approved by the local ethics committee. Subjects practiced recreational sports, but did not participate in any strength or flexibility training at the time of the study. No subjects had sustained a recent injury that may have affected the findings.

The experimental design has been previously described 10,11 and has been adapted from previous studies. ${ }^{6,7,13}$ Briefly, the Biodex system 3 research $^{\circledR}$ isokinetic dynamometer (Biodex medical, Shirley, NY, USA) was used to measure torque produced in resistance to passive stretch $(T)$, knee joint angle $(\theta)$ and knee joint angular velocity $(\omega)$. Subjects were seated and the thigh was fastened using Velcro straps to a thigh pad elevating it from horizontal. The trunk-thigh angle was adjusted to $60^{\circ}$, and the input axis of the dynamometer was aligned with the approximate axis of rotation of the knee joint. This position pre-tensioned the hamstring muscles and all subjects were unable to reach full knee extension from this position. All procedures began with the lower leg perpendicular to the thigh. This position was used to determine our reference knee angle expressed as $0^{\circ} . T, \theta$ and $\omega$ were sampled at $256 \mathrm{~Hz}$ with a 12 bits A/D converter (Myodata, Electronique du Mazet, Le Mazet, France). Data were stored in a flash memory card (20 Mo) and transferred to a computer hard disk for further analysis.

All subjects performed two sessions that were separated by one day. First, a familiarisation session was performed in order to prepare the subjects for all testing procedures. During the main session, a baseline-test was followed by a static stretching protocol, and thereafter a post test was performed. Baseline and post tests were similar and included the following: (i) a maximal knee range of motion (ROM) measurement. In this test, the right lower leg was passively extended $\left(\omega=5^{\circ} \mathrm{s}^{-1}\right)$, and the subjects used a stop switch when they perceived the maximum tolerable hamstring muscle stretch. This point was operationally defined as their maximal range of motion (ROM). The leg was then immediately returned to the starting position; (ii) five cyclic $\left(\omega=5^{\circ} \mathrm{s}^{-1}\right)$ passive repetitions at $80 \%$ of the ROM measured during the baseline-test.

The stretching protocol was composed of six $30 \mathrm{~s}$ - static hamstring stretches. Specifically, the right leg was passively extended to a predetermined knee angle $\left(\omega=5^{\circ} \mathrm{s}^{-1}\right)$, maintained for $30 \mathrm{~s}$ in this position and unloaded to the initial position $\left(\omega=5^{\circ} \mathrm{s}^{-1}\right)$. The predetermined knee angle was $80 \%$ of the pre-test ROM for the first stretching repetition. It was then increased with a $2 \%$ step between each repetition until $90 \%$ of the ROM for the sixth repetition. No rest period was provided between each stretching repetition. To avoid any effects of the baseline-test, a $15 \mathrm{~min}$ rest period was observed before the static stretching protocol. ${ }^{6}$ No rest period was observed between the static stretching protocol being completed and the post test.

Surface electromyographic signals (sEMG) of the hamstring muscles were also recorded synchronously with the torque and angle data to ensure that no undesirable activation occurred during the stretches. Bipolar surface electromyographic (sEMG) signals were recorded from surface electrodes $(\mathrm{Ag} / \mathrm{AgCl}, 4 \mathrm{~mm}$ recording diameter, In Vivo Metric, Healdsburg, CA, USA) placed on semitendinosus and biceps femoris muscles with an $11 \mathrm{~mm}$ inter-electrode distance according to the surface electromyography for the non-invasive assessment of muscles (SENIAM) recommendations. ${ }^{14}$ sEMG signals were sampled at $1024 \mathrm{~Hz}$ using the same A/D converter (Myodata, Electronique du Mazet, Le Mazet, France) utilised for the mechanical signals. In order to normalise the sEMG data recorded during the passive stretching trials, sEMG data were collected during three maximal effort knee flexion and extension repetitions undertaken at an angular velocity of $60^{\circ} \mathrm{s}^{-1}$. Stretching trials in which normalised sEMG activity levels were higher than $1.5 \%$ were discarded. ${ }^{11}$

All the data were processed using a standardised program computed with Matlab ${ }^{\circledR}$ (The Mathworks, Natick, USA). Mechanical signals ( $T, \theta$ and $\omega$ ) were filtered using a Butterworth second order low pass filter $(10 \mathrm{~Hz})$. Recorded torque was corrected for the limb mass. ${ }^{15}$ The $T-\theta$ relationships were then fitted using a modified Sten-Knudsen model ${ }^{16}$ which has been shown to be appropriate for our analyses ${ }^{10}$ :

$\left(\frac{A}{\alpha}\right)\left(\mathrm{e}^{\alpha \theta}-B\right)$

where $A, B$ and $\alpha$ are experimental constants.

Thereafter, using a similar method to Weir et al., ${ }^{12}$ the difference in angle $\left(\Delta \theta_{i}\right)$ between pre and post stretching torque-angle curves was calculated at 11 levels of torque from $0 \%$ to $100 \%$ ( $10 \%$ increments) of the maximal torque generated during the passive motion (see Fig. 1). The mean change in angle $(\Delta \theta)$ across these 11 torque levels was then 


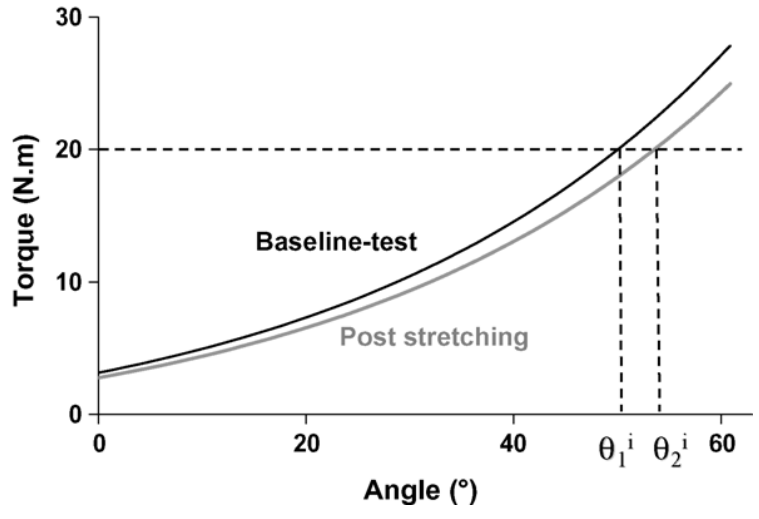

Fig. 1. A schematic of passive torque and angle illustrating the method to calculate the change in angle between curves. This procedure was repeated for eleven equidistant torque levels (every 10\%) of torque. Shifts of the torque-angle relationships $(\Delta \theta)$ corresponded to the average of the 11 differences between $\theta_{2}^{i}$ and $\theta_{1}^{i}$.

calculated. This procedure was also undertaken for a comparison of the first and fifth cycle of the cyclic stretching.

After checking the distribution of data (Kolmogorov -Smirnov test), parametric statistical tests were performed using Statistica ${ }^{\circledR}$ software. Two $(1 \times 11)$ repeated measures analyses of variance (ANOVAs) were used to determine changes in $\Delta \theta_{i}$ across the 11 levels of torque for the effects of static and cyclic stretching. One $(2 \times 3)$ ANOVA was used to compare $\Delta \theta_{i}$ changes after cyclic and static stretching across the 3 levels of torque $(0 \%, 50 \%$ and $100 \%$ of the maximal passive torque). Newman-Keuls post hoc analysis was used when appropriate. The critical level of significance in the present study was set at $P<0.05$.

\section{Results}

The passive torque was decreased after static $(-1.8 \pm 1.6 \mathrm{Nm}, \quad$ Fig. 2A) and cyclic stretching $(-2.5 \pm 1.5 \mathrm{Nm}$, Fig. 2B) protocols. No significant main effect $(P>0.05)$ was found for $\Delta \theta_{i}$ across torque levels after static stretching indicating that difference in angle was constant (Fig. 3A). The mean $\Delta \theta$ post static stretching cycle was $5.2 \pm 4.6^{\circ}\left(0.6-13.7^{\circ}\right)$. A significant main effect $(P<0.001)$ was found for $\Delta \theta_{i}$ after cyclic stretching across torque levels indicating that the angle change was not constant across the range of torque (Fig. 3B). At the start of motion, the angle change was $11.1 \pm 2.6^{\circ}$, and it decreased linearly to $1.6 \pm 1.6^{\circ}$ at the most extended position. The mean $\Delta \theta$ was similar to that of static stretching $5.5 \pm 2.2^{\circ}$ $\left(1.61-7.97^{\circ}\right)$. No significant main effect for stretching mode was found for $\Delta \theta_{i}$, while main effect for the torque level $(P<0.001)$ and interaction (stretching mode $\times$ torque level) were significant (Fig. $4, P<0.001$ ). $\Delta \theta_{i}$ was significantly different across the three torque levels after cyclic stretching $(P<0.01)$, while no significant difference was found after static stretching $(P>0.05)$.
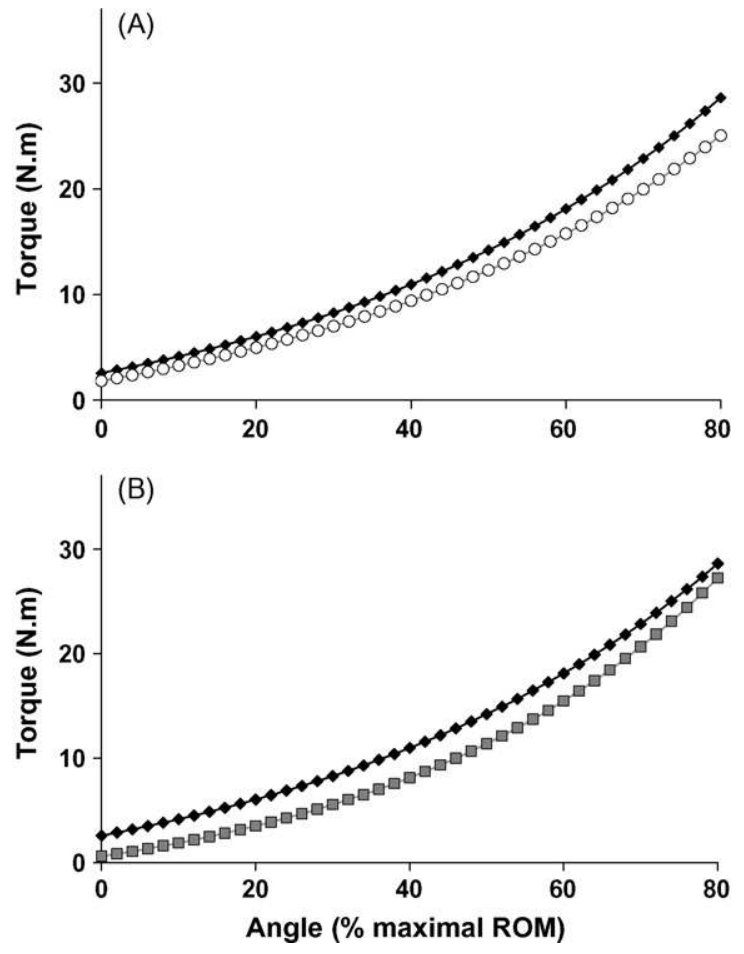

Fig. 2. Averaged relationships between the passive torque and the angle (expressed in percentage of the baseline maximal range of motion, ROM). Error bars are removed for clarity. (A) Relationships of the baseline ( $)$ and the post static stretching test $(\bigcirc)$. (B) Relationships of the baseline $(\checkmark)$ and the fifth cycle ( $\square)$.
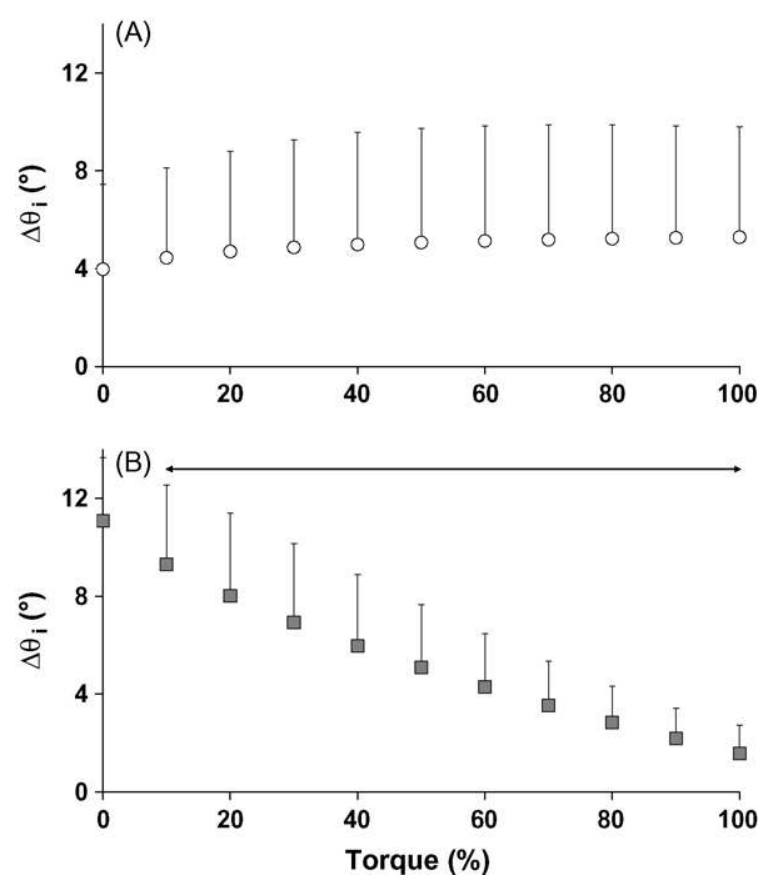

Fig. 3. Differences in angle $\left(\Delta \theta_{i}\right)$ at 11 levels of torque (expressed in percentage of the maximal torque) (A) between pre and post stretching torque-angle curves (effect of static stretching); (B) between first and fifth cycles torque-angle curves (effects of cyclic stretching). $\leftrightarrow$ : significant difference $(P<0.01)$ with the value at $0 \%$ of torque. 


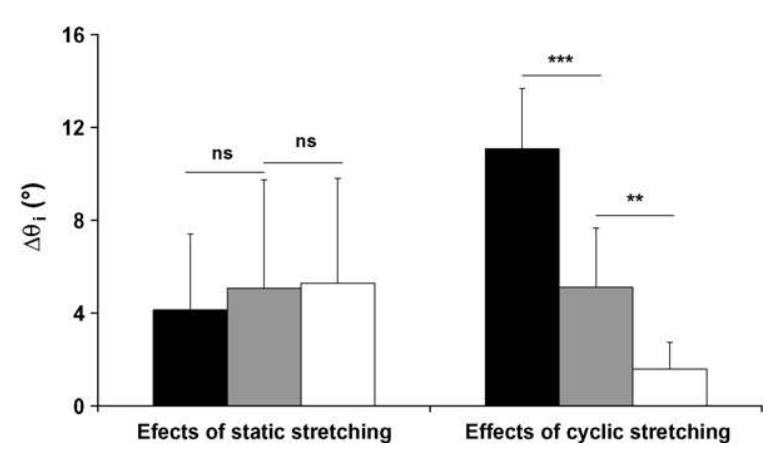

Fig. 4. Changes in angle $\left(\Delta \theta_{i}\right)$ at 3 levels of torque $(0 \%$ of the maximal torque level in black, $50 \%$ in grey and $100 \%$ in white) after static and cyclic stretching protocols. ns: non significant, ${ }^{* *} P<0.01 ;{ }^{* * *} P<0.001$

\section{Discussion}

While previous work has focused upon measurements of single angles to illustrate change in the torque-angle relationship, the current study was designed to assess the effect of cyclic and static stretching protocols across the whole passive torque-angle relationship. No previous work has compared cyclic and static stretching in this manner. After cyclic stretching, the different changes in angle across torque levels (Fig. 3B) indicated that the shape of the torque-angle relationship was altered. Our results demonstrated that following the static stretching protocol a mean constant change in passive torque-angle curve of $5.2^{\circ}$ was observed. Since the angle changes were constant across torque levels (Fig. 3A) this finding provided evidence that the shape of the passive torque-angle curve was unchanged after static stretching.

The latter finding suggests that dissipative properties are not greatly influenced by static stretching and provides some evidence that viscous effects are unlikely to be playing a significant role. ${ }^{11}$ However, this finding might be expected with changes in muscle resting length. In isolated muscle, acute increases in muscle resting length have been demonstrated in rabbit muscle after $10 \times 30 \mathrm{~s}$ of static stretching. ${ }^{17}$ Taylor et al. ${ }^{17}$ suggested that they could be due to creep in the muscle-tendon unit and our results confirm this hypothesis for an in vivo protocol. To our knowledge, only Yeh et al. ${ }^{18}$ have performed a creep experiment on a passive musculo-articular complex in vivo. These authors examined the plantar flexors of subjects with spasticity and showed that $30 \mathrm{~min}$ of continuous stretching of plantar flexors at a constant torque level induced an acute increase in joint angle of approximately $4^{\circ}$. The creep response might be due to different mechanical processes taking place within microstructures of both muscle and tendon tissues but the relative contribution of these structures to the lengthening is not yet known.

Since the shape of the torque-angle relationship was altered after cyclic stretching, the mechanisms involved in cyclic and static stretching protocols are at least in part different, and this difference may reflect changes in dissipative properties. In support of this conjecture, Nordez et al. ${ }^{11}$ showed that cyclic stretching induced a decrease in passive torque during loading primarily at the beginning of the range of motion. In contrast the unloading curve was unchanged. Consequently, the energy stored during the loading (i.e. the area under the loading torque-angle relationship) was decreased, while the energy restituted (i.e. the area under the unloading torque-angle relationship) was unchanged. Thus, the dissipation coefficient (DC), calculated as the energy dissipated normalised by the energy store ${ }^{11}$ was decreased after cyclic stretching. The decrease in DC can be interpreted as a decrease in viscosity. ${ }^{19}$ As such, the musculo-articular complex displays thixotropic behavior. Three mechanisms might be responsible for these changes: (i) it has been shown on isolated muscle that stable bonds between actin and myosin filaments contribute to the muscle passive tension and that these bonds are broken by stretching the muscle. ${ }^{20,21}$ However, in respect to the stretching protocol in the current study, recent research ${ }^{22}$ has shown that these effects are probably negligible; (ii) it has also been proposed that the more mobile constituent of muscles (e.g. polysaccharides and water) might be redistributed during stretching and that this change in the structural arrangement of muscle could explain the thixotropy of the musculo-articular complex ${ }^{8}$; (iii) collagen may exhibit a thixotropic behavior, through the rearrangement/slipping of fibers during stretching. ${ }^{23,24}$

Whether the current findings have ramifications for performance is difficult to appreciate. The results of numerous studies $^{25-28}$ indicate a significant decrease in muscle torque production following stretching. However, these have generally been assessed at a single angle. The current findings indicate that following static stretching, changes are apparent across the full range of motion and therefore it could be hypothesised that this type of stretching may have more influence upon performance in sporting activities where force is required across the range of motion. These thoughts are speculative and further work focused upon this area is needed.

\section{Conclusion}

Different effects were observed in the passive torqueangle curve after acute bouts of cyclic and static stretching. While the static stretching protocol led to a constant change in range of motion across torque levels and the passive torque-angle curve retained its baseline shape, cyclic stretching led to a change in the shape of the passive torque-angle curve. The former finding provided some evidence that the decrease in passive torque following static stretching could be explained primarily by acute increases in muscle resting length while the latter was suggestive of a mechanism involving the dissipative properties of the muscles, and it was proposed that thixotropy was implicated. Further research is needed to have a better understanding of thixotropy, which is known to be one of the more complex mechanical behaviors of a material. 


\section{Practical implications}

- Our results, show that the effects of cyclic and static stretching on passive torque-angle curves are different.

- Findings of the current study indicate that following static stretching, changes are apparent across the full range of motion and the constant shift to the right of the passive torque-angle relationship indicates that an acute increase in muscle resting length may have occurred. This increase could be more beneficial for improving flexibility, but could also affect the active force-length relationship. Therefore, it could be hypothesised that this type of stretching may have more influence upon performance in sporting activities where force is required across the range of motion and hence be more detrimental to performance than cyclic stretching.

\section{Conflict of interest}

All authors agree that there are no conflict of interest issues in this research.

\section{References}

1. Riemann BL, DeMont RG, Ryu K, et al. The effects of sex, joint angle, and the gastrocnemius muscle on passive ankle joint complex stiffness. J Athl Train 2001;36(4):369-75.

2. Gajdosik RL. Passive extensibility of skeletal muscle: review of the literature with clinical implications. Clin Biomech 2001;16(16):87-101.

3. Gajdosik RL, Vander Linden DW, McNair PJ, et al. Viscoelastic properties of short calf muscle-tendon units of older women: effects of slow and fast passive dorsiflexion stretches in vivo. Eur J Appl Physiol 2005;95(2-3):131-9.

4. Gajdosik RL, Vander Linden DW, McNair PJ, et al. Effects of an eight-week stretching program on the passive-elastic properties and function of the calf muscles of older women. Clin Biomech (Bristol, Avon) 2005;20(9):973-83.

5. Magnusson SP. Passive properties of human skeletal muscle during stretch maneuvers. A review. Scand J Med Sci Sports 1998;8(2):65-77.

6. Magnusson SP, Aagard P, Simonsen E, et al. A biomechanical evaluation of cyclic and static stretch in human skeletal muscle. Int $J$ Sports Med 1998;19(5):310-6.

7. Magnusson SP, Simonsen EB, Aagaard P, et al. Biomechanical responses to repeated stretches in human hamstring muscle in vivo. Am J Sports Med 1996;24(5):622-8.

8. McNair PJ, Dombroski EW, Hewson DJ, et al. Stretching at the ankle joint: viscoelastic responses to holds and continuous passive motion. Med Sci Sports Exerc 2001;33(3):354-8.

9. McNair PJ, Hewson DJ, Dombroski E, et al. Stiffness and passive peak force changes at the ankle joint: the effect of different joint angular velocities. Clin Biomech (Bristol, Avon) 2002;17(7):536-40.
10. Nordez A, Cornu C, McNair P. Acute effects of static stretching on passive stiffness of the hamstring muscles calculated using different mathematical models. Clin Biomech 2006;21:755-60.

11. Nordez A, McNair PJ, Casari P, et al. Acute changes in hamstrings musculo-articular dissipative properties induced by cyclic and static stretching. Int J Sports Med 2008;29(5):414-8.

12. Weir DE, Tingley J, Elder GC. Acute passive stretching alters the mechanical properties of human plantar flexors and the optimal angle for maximal voluntary contraction. Eur J Appl Physiol 2005;93(5-6):614-23.

13. Reid DA, McNair PJ. Passive force, angle, and stiffness changes after stretching of hamstring muscles. Med Sci Sports Exerc 2004;36(11): 1944-8.

14. Hermens HJ, Freriks B, Disselhorst-Klug C, et al. Development of recommendations for SEMG sensors and sensor placement procedures. J Electromyogr Kinesiol 2000;10:361-74.

15. Aagaard P, Simonsen EB, Trolle $M$, et al. Isokinetic hamstring/quadriceps strength ratio: influence from joint angular velocity, gravity correction and contraction mode. Acta Physiol Scand 1995; 154:421-7.

16. Sten-Knudsen O. Torsional elsticity of the isolated cross-striated muscle fibre. Acta Physiol Scand 1953;28:1-240.

17. Taylor CD, Dalton JD, Seaber AV, et al. Viscoelastic properties of muscle-tendon units. The biomechanical effetcts of stretching. Am J Sports Med 1990;18(3):300-9.

18. Yeh CY, Tsai KH, Chen JJ. Effects of prolonged muscle stretching with constant torque or constant angle on hypertonic calf muscles. Arch Phys Med Rehabil 2005;86(2):235-41.

19. Mandel J. Aperçu sur les principaux comportements rhélogiques. In: Persoz B, editor. La rhéololgie. Paris: Masson \& Cie; 1969. p. $1-17$.

20. Proske U, Morgan DL. Do cross-bridges contribute to the tension during stretch of passive muscle? J Muscle Res Cell Motil 1999;20: 433-44.

21. Whitehead NP, Gregory JE, Morgan DL, et al. Passive mechanical properties of the medial gastrocnemius muscle of the cat. $J$ Physiol 2001;536:893-903.

22. Morse CI, Degens H, Seynnes OR, et al. The acute effect of stretching on the passive stiffness of the human gastrocnemius muscle tendon unit. J Physiol 2008;586(Pt 1):97-106.

23. Kubo K, Kanehisa H, Kawakami Y, et al. Influence of static stretching on viscoelastic properties of human tendon structures in vivo. J Appl Physiol 2001;90(2):520-7.

24. Stromberg DD, Wiederielm CA. Viscoelastic description of a collagenous tissue in simple elongation. J Appl Physiol 1969;26: $857-62$

25. Cramer JT, Housh TJ, Weir JP, et al. The acute effects of static stretching on peak torque, mean power output, electromyography, and mechanomyography. Eur J Appl Physiol 2005;93(5-6):530-9.

26. Shrier I. Does stretching improve performance? A systematic and critical review of the literature. Clin J Sport Med 2004;14(5): 267-73.

27. Nelson AG, Guillory IK, Cornwell C, et al. Inhibition of maximal voluntary isokinetic torque production following stretching is velocityspecific. J Strength Cond Res 2001;15(2):241-6.

28. Fowles JR, Sale DG, MacDougall JD. Reduced strength after passive stretch of the human plantarflexors. J Appl Physiol 2000;89(3): 1179-88. 